\title{
Impact of Converter Losses on the Optimal Power Flow solution of Hybrid Networks based on VSC-MTDC
}

\author{
Q. Zhao ${ }^{\mathrm{a}, *}$, J. García-González ${ }^{\mathrm{a}}$, O. Gomis-Bellmunt ${ }^{\mathrm{b}}$, E. Prieto-Araujo $^{\mathrm{b}}, \mathrm{F}$. \\ M. Echavarren ${ }^{\mathrm{a}}$ \\ ${ }^{a}$ Institute for Research in Technology (IIT), ICAI School of Engineering, Comillas \\ Pontifical University, Madrid, Spain \\ ${ }^{b}$ CITCEA, Electrical Engineering Department, Technical University of Catalonia, \\ Barcelona, Spain
}

\begin{abstract}
This paper studies the impact of Voltage Source Converter (VSC) losses on the solution of the Optimal Power Flow (OPF) of hybrid AC/DC systems with a multi-terminal configuration. The motivation of this analysis is that the expected development of High Voltage Direct Current (HVDC) systems will entail an increase of the number of converter stations, and consequently, converter losses might not be negligible when compared with conventional transmission losses of AC and DC lines. Towards this end, an extended OPF model is proposed considering a combination of VSC based Multi-Terminal HVDC grids (VSC-MTDC) and AC systems. The OPF model represents converter losses according to the state-of-the art modeling where different expressions are used when the converter functions as an inverter or as a rectifier. Three simpler alternative approaches are also implemented and the obtained OPF solutions are assessed. In order to compare the OPF solutions, a comparison metric is proposed. Obtained results show that modeling the converter losses in a simplified way could lead to very different power flow solutions, especially for the DC branches.
\end{abstract}

Keywords: Optimal Power Flow, Multi-Terminal HVDC, AC/DC

Transmission System, Cost Minimization, Converter Losses

\section{Nomenclature}

Upper-case letters are used for denoting parameters and sets. Lower-case letters denote variables and indexes. The superscripts " $a c$ " and " $d c$ " are used to denote AC and DC variables respectively, among which those that are in bold indicate vectors or matrices.

\footnotetext{
* Corresponding author

Email address: quanyu.zhao@comillas.edu (Q. Zhao)
} 


\subsection{Indexes, sets and subsets}

$g \in \mathcal{G} \quad$ Generating units, running from 1 to $G$

$i, j \in \mathcal{B} \quad$ Buses, running from 1 to $B$

$l \in \mathcal{L} \quad$ Transmission branches, including lines and transformers, running from 1 to $L$

$v \in \mathcal{V} \quad$ VSC converter, running from 1 to $V$

$(i, j) \in \mathcal{N}_{l}$ Pair of buses connected by line $l$

$\mathcal{L}^{a c} \subset \mathcal{L} \quad$ Subset of AC transmission lines

$\mathcal{L}^{d c} \subset \mathcal{L} \quad$ Subset of DC transmission lines

$\mathcal{B}^{a c} \subset \mathcal{B} \quad$ Subset of AC buses

$\mathcal{B}^{d c} \subset \mathcal{B} \quad$ Subset of DC buses

$\mathcal{N}_{v}^{a c} \quad$ AC bus of converter $v$

$\mathcal{N}_{v}^{d c} \quad$ DC bus of converter $v$

\subsection{Parameters}

$R_{l} \quad$ Resistance of line $l$

$G_{l} \quad$ Conductance of line $l$

$B_{l} \quad$ Susceptance of line $l$

$B_{l}^{\text {sht }} \quad$ Half total line charging susceptance of line $l$

$A_{g}, B_{g}, C_{g}$ Cost coefficients of generator

$N C_{p}, N C_{q}$ Costs of non-served real and reactive power

$A_{v}, B_{v} \quad$ Independent and linear terms of the losses function of converter $v$

$C_{v}^{r e c}, C_{v}^{i n v}$ Quadratic terms of the losses function of converter $v$ acting as rectifier/inverter

$\bar{S}_{l}, \bar{P}_{l} \quad$ Flow limits on transmission line $l$

$\bar{I}_{v} \quad$ Maximum current allowed through the converter $v$

$\bar{P}_{g}, \underline{P}_{g} \quad$ Real power limits of generator $g$

$\bar{Q}_{g}, \underline{Q}_{g} \quad$ Reactive power limits of generator $g$

$\bar{V}_{i}, \underline{V}_{i} \quad$ Voltage limits at bus $i$.

$P_{D i}, Q_{D i} \quad$ Real and reactive power demand at bus $i$. 


$\begin{array}{ll}\mathbf{G}_{\mathbf{i j}}^{\mathbf{a c}} & \text { AC network conductance matrix } \\ \mathbf{G}_{\mathbf{i j}}^{\mathbf{d c}} & \text { DC network conductance matrix } \\ \mathbf{B}_{\mathbf{i j}} & \text { AC network susceptance matrix } \\ \text { 0.3. Decision Variables } \\ p_{i}, q_{i} & \text { Real and reactive power injected at bus } i \\ \rho_{v}, \rho_{l} & \text { Active power losses of converter } v \text { and line } l \\ n p_{i}, n q_{i} & \text { Non-served active and reactive power at bus } i \\ v_{i}, \theta_{i} & \text { Voltage magnitude and phase angle at bus } i \\ p_{g}, q_{g} & \text { Active and reactive power generation of generator } g \\ i_{v}^{i n v}, i_{v}^{r e c} & \text { Module of phase current of converter } v \text { when functions as an inverter } \\ & \text { and rectifier respectively }\end{array}$

\section{Introduction}

Meeting the rising energy requirements in a sustainable, secure and competitive manner is one of the main challenges of current power systems. In this context, the development of a "Supergrid" has been proposed as a promising solution to harness geographically dispersed low-carbon energy sources (such as offshore wind and solar), and also to facilitate the cross-border trading and the integration of wholesale electricity markets [1]. For instance, in the European system, $150 \mathrm{GW}$ of total installed offshore capacity of wind energy producing $562 \mathrm{TWh}$ of electricity is expected by 2030 [2], and increasing the capacity of the transnational interconnections is in the agenda of the current EU energy policy [3].

Despite the existence of many barriers such as the cost of converter stations, less standardized equipment as compared with AC systems, need of new control algorithms, difficulty to build DC breakers, etc., High Voltage Direct 
Current (HVDC) systems with Multi-Terminal (MTDC) configuration are seen as a viable option that can outperform traditional $\mathrm{AC}$ transmission due to its technical, economic and environmental advantages [4].

Compared to Current Source Converter (CSC), Voltage Source Converter (VSC) offers some great advantages [5]. Due to completely different operating principles, new algorithms have been developed for VSC HVDC control and Power Flow (PF) studies [6]. The problem of finding the PF solution for the case of a hybrid network with VSC-MTDC systems is relatively new [7], [8]. The Optimal Power Flow (OPF) problem for hybrid networks is even a less developed research line, and the converter's operation introduces additional decision variables that increase the complexity of the resulting optimization problem, [8], [9], [10].

In case of a large deployment of HVDC networks, the impact of their converter stations on the control, operation and planning of the whole power system needs to be carefully examined. For instance, the ratio between the voltage levels at the AC and DC sides of the converter is limited due to the constraints imposed by the power electronic equipment. In addition, the amount of active and reactive power injected or withdrawn at the $\mathrm{AC}$ node has to respect the P-Q capability curves. Among the converter characteristics, this paper focuses on the effect of the losses incurred during the converter operation. As in any energy transformation process, the converter operation is not $100 \%$ efficient, and therefore, there will be always a difference between the active power injected at one terminal, and the active power withdrawn at the other. Typical values of such losses ranges from $1 \%$ up to $3 \%$ of the total power going through the converter [7]. Therefore, for a hypothetical case of a large HVDC Supergrid, the active power losses of the converters could represent a significant portion of all the system losses. To the author's knowledge, there are no previous studies 
that have assessed the impact of converter losses on the solution of the OPF for hybrid networks. From the optimal operation point of view, it is common to model $\mathrm{AC}$ and $\mathrm{DC}$ transmission losses so that the $\mathrm{OPF}$ solution takes into account them when deciding the optimal generators scheduling. Due to the non-linear nature of the power flow equations, such transmission losses are in many cases approximated by means of simplified formulations (for instance as piece-wise linear functions [11]). The proper modeling of converter losses is neither a simple task as they are caused by several reasons: ohmic losses at the cables, switching of the semiconductors, etc. In this paper, the theoretical converter losses will be computed as a polynomial function that depends on the phase current of the converter, and taking into account that losses can be different when the converter acts as an inverter or as a rectifier (i.e. active power injected at the AC bus or at the DC bus respectively). Given that such detailed modeling of converter losses could lead to a heavy computational burden, this paper will also analyze the impact of some alternative ways of modeling the converter losses in a more simplified manner. In this sense, conventional DC and $\mathrm{AC}$ transmission losses will be modeled by means of the exact power flow equations, so that the obtained results allow isolating the effect of the approach followed to model converter losses.

The main contributions of this paper are the following ones:

1. The first contribution is the proposed methodology to assess the effect of the different approaches to model converter losses on the solution of the OPF. This methodology includes the definition of a metric used for the required comparisons. To authors' knowledge, the topic addressed in this paper has not been studied previously, and the selected study cases with different HVDC topologies highlight the importance of a proper converter losses representation. 
2. The OPF model used in this assessment constitutes the second main contribution of this paper. The developed model takes as starting point the model presented in [10], which has been improved in order to model the converter losses by means of the most accurate representation found in PF studies.

This paper is organized as follows. VSC modeling is described in detail in Section 2. In Section 3, equations used for modeling a hybrid network are presented. Proposed methodology to assess impact of converter losses is illustrated in Section 4. The results and discussions of the case study are presented in Section 5 . Finally, conclusions are drawn in Section 6.

\section{Voltage Source Converter (VSC)}

\subsection{Types of $V S C$}

The VSC station comprises all the elements that connect the AC and the DC networks. Each VSC will be referred to with the index $v$. At the converter, the voltage waveform is synthesized either by Pulse Width Modulation (PWM) that requires low pass filters to block the flow of higher order harmonics, or by a Modular Multilevel Converter (MMC) approach.

VSC typically use Insulated-Gate Bipolar Transistors (IGBTs), enabling a controlled two or three-level voltage output driven by Pulse Width Modulation (PWM) [12]. This is not well suited for high voltage applications as IGBTs can only withstand a few kilovolts. There are some proposals to chain several IGBTs to create high voltage switches, requiring a sophisticated driver circuit, difficult to scale for high voltages. Comparing to MMC, PWM-based HVDC converters present higher losses, due to the high switching frequency. MMCs structure is able to operate at large voltages, combining a large number of controlled 


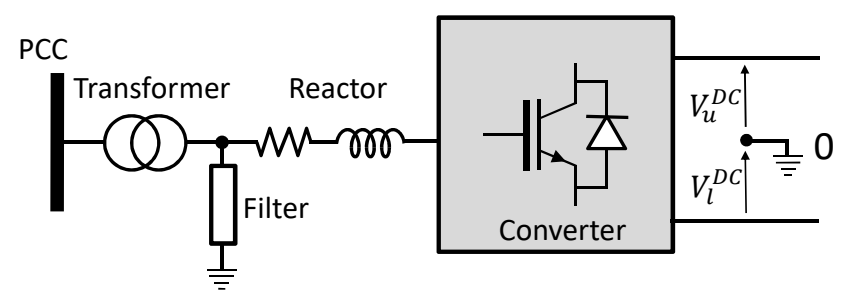

Figure 1: Three phase scheme and single-phase diagram of the VSC

submodules (SM), while reducing the switching frequency and consequently resulting in less losses. Moreover, MMCs have several additional benefits such as reduced harmonic content, reduced transformer $\mathrm{dv} / \mathrm{dt}$ stress and a great potential for standardization. Therefore, MMC is foreseen as the technology of choice for VSC HVDC transmission. Nevertheless, many VSCs nowadays use PWM based on two or three-level topologies [7]. Without loss of generality, the stateof-the-art approach is adopted in this paper for converter modeling regardless of converter type, which will be explained further in detail.

The filter bus is connected to the AC network through a transformer and the power can flow in both directions, (see Fig. 1). When the active power is taken from the $\mathrm{AC}$ side and injected at the $\mathrm{DC}$ side, the converter is said to be operated as a rectifier. Otherwise, the converter is operated as an inverter. The converter can also inject or absorb reactive power from the AC side. The variables (in p.u.) that represent the active and reactive power injected to the AC side of the converter $\nu$ are defined as $p_{v}^{a c}$ and $q_{v}^{a c}$. These variables can be either positive or negative depending on the operation mode of the converter. The same applies to the power injected to the DC side of the converter, i.e. $p_{v}^{d c}$.

\subsection{Converter Losses}

During the conversion process, the available real power at one side of the converter will be lower than the active power injected at the other side due to the converter losses, $\rho_{v}$. 
There are several source of losses within an MMC, such as semiconductor losses in each of the SMs, arm reactor losses, phase reactor losses or transformer losses. Focusing on the semiconductor losses, two different types can also be distinguished, the switching and the conduction losses [13]. Several publications show different methods addressing the losses calculations of and MMC [13], [14], [15], [16]. Similarly, several components contribute to the losses of PWM-based converters which can be potentially complex as well [7]. However, such detailed procedures cannot be included directly in an optimization model. For this reason, a polynomial expression is adopted in this paper, as it is conventionally used in the PF state-of-the-art models [6]. In particular, converter losses will be expressed as a quadratic function that depends on the value and the direction of the phase current of the converter. Following the same criteria as above for the active power, the phase current $i_{v}$ will take positive values when the converter injects it at the $\mathrm{AC}$ bus (inverter), or as negative in the opposite case (rectifier). Therefore, the mathematical expression of the losses that will be used as benchmark values for the comparison ("Complete" modeling) is the one shown in (1):

$$
\rho_{v}=A_{v}+B_{v} \cdot\left|i_{v}\right|+C_{v}^{i n v} \cdot\left(i_{v}^{i n v}\right)^{2}+C_{v}^{r e c} \cdot\left(i_{v}^{r e c}\right)^{2}
$$

where $i_{v}=i_{v}^{i n v}-i_{v}^{r e c}$ and $i_{v}^{i n v}, i_{v}^{r e c} \geq 0 ; A_{v}, B_{v}$ and $C_{v}^{r e c}, C_{v}^{i n v}$ are corresponding converter loss coefficients. Adding as an extra condition that only one of them can be different to zero, only one of the quadratic terms $C_{v}^{i n v} \cdot\left(i_{v}^{i n v}\right)^{2}$ or $C_{v}^{r e c} \cdot\left(i_{v}^{r e c}\right)^{2}$ will be activated. In addition, the absolute value used in the linear term could be computed as $\left|i_{v}\right|=i_{v}^{i n v}+i_{v}^{r e c}$.

Furthermore, all converter losses are allocated according to the same criterion used in the software MatACDC, as that will be the validation tool used in this paper to check the correctness of the obtained power flows with the proposed OPF model. 
The state-of-the-art modeling of converter losses for steady-state power flow analysis is the one presented in [6], and later on included in the CIGRE report [17]. In that work, the authors differentiate between the operation of the converter as a rectifier or as an inverter, and therefore, the coefficients of the polynomials used to model the losses can be different depending on the direction of the active power transferred. In a power flow model, the converter operation mode (inverter or rectifier) must be known in advance. By contrast, in the "Complete" OPF model presented in this paper, the operation mode is a decision variable, and the optimization problem determines the optimal operation of every converter taking into account the very same representation of the losses as the one presented in [6].

In order to assess the impact of converter losses on the OPF solution, we have proposed other three simplified approaches: "average" modeling which does not differentiate between inverter/rectifier modes; "proportional" modeling which assumes converter losses are proportional to the real power injected from the converter into the $\mathrm{AC}$ side; and finally "lossless" modeling that neglects converter losses.

\subsubsection{Average modeling ("Avg")}

It models the converter losses without differentiating between the inverter/rectifier modes by selecting the quadratic coefficient equal to the average of $C_{v}^{i n v}$ and $C_{v}^{r e c}$, i.e., $C_{v}^{a v g}=\left(C_{v}^{i n v}+C_{v}^{r e c}\right) / 2$, as shown below.

$$
\rho_{v}=A_{v}+B_{v} \cdot\left|i_{v}\right|+C_{v}^{a v g} \cdot\left|i_{v}\right|^{2}
$$

\subsubsection{Proportional modeling ("Prop")}

In this case, the converter losses are assumed to be proportional to the absolute value of the real power $p_{c}$ (see Fig. 2) injected from the converter to the $c$ node on the AC side with certain ratio $\alpha . \rho_{v}$ is depicted in Fig. 3 and 
explained with (4). The constraint thereby can be written as follows:

$$
\rho_{v}=\alpha \cdot\left|p_{c}\right|
$$

\subsubsection{Lossless modeling ("Lss")}

This model simply assumes a lossless converter, thus only transmission losses (AC and DC lines) are considered.

\subsection{Equivalent circuit of VSC}

As described in [6], [10], the most common approach is to represent the VSC converter as a controllable voltage source $\boldsymbol{v}_{\boldsymbol{c}}=v_{c} \angle \theta_{c}$ connected by a phase reactor $\boldsymbol{z}_{c}=r_{c}+j x_{c}$ to an intermediate node where a lossless shunt filter is connected $\left(\boldsymbol{z}_{f}=-j / b_{f}\right)$. The voltage at this intermediate node is $v_{f} \angle \theta_{f}$, and the transformer that be represented by its impedance: $\boldsymbol{z}_{t f}=r_{t f}+j x_{t f}$ (see Fig. 2).

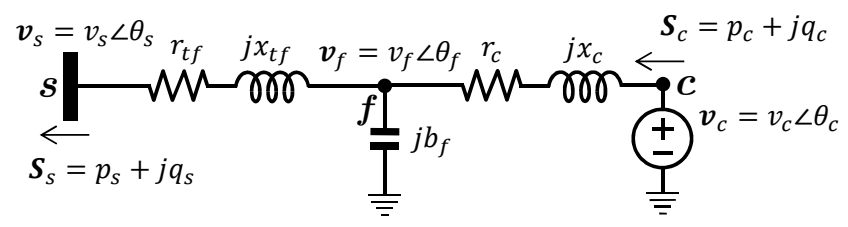

Figure 2: Equivalent circuit of the VSC-Converter Station

It is important to notice that for each VSC converter station, two more $\mathrm{AC}$ buses are added to the system: the filter bus (voltage $v_{f} \angle \theta_{f}$ ), and the converter bus (voltage $v_{c} \angle \theta_{c}$ ). In case of not being necessary to install the filter (or when its effect can be neglected), both the phase reactor and the transformer impedance can be lumped together, eliminating from the equations the corresponding voltage magnitude and phase angle of the filter bus. As a consequence, the power flow within the VSC converter station between the nodes $c, f$, and $s$ have to comply with the standard AC power flow equations. In that 


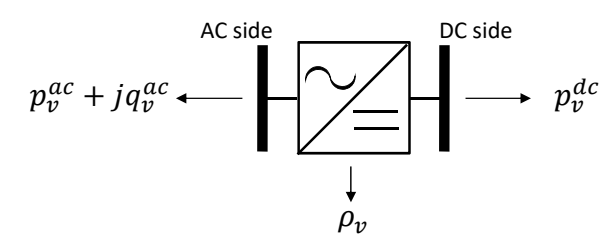

Figure 3: Power balance at the VSC converter

case, the shunt susceptance only affects the diagonal terms of the matrix $\mathbf{B}$ at the position of the filter buses. Depending on the level of accuracy (transformeror filter-less), converter power injection to the $\mathrm{AC}$ network $p_{v}^{a c}+j q_{v}^{a c}$ can be treated equivalent as $\boldsymbol{s}_{\boldsymbol{c}}=p_{c}+j q_{c}$ or $\boldsymbol{s}_{\boldsymbol{s}}=p_{s}+j q_{s}$.

Regarding the power balance at the converter, Fig. 3 shows the criterion used in this paper where the arrows indicate when the injected power is considered positive. The corresponding active power balance equation is established in (4), where power losses at the converter can take only positive values, i.e., $\rho_{v} \geq 0$.

$$
0=p_{v}^{d c}+p_{v}^{a c}+\rho_{v}, \forall v \in \mathcal{V}
$$

\subsection{VSC operation limits}

There are mainly three factors limiting the operation of VSC-based HVDC systems [9], which are described next.

\subsubsection{Maximum current through the IGBTs}

This limit is meant to safeguard the switching elements of the VSC as the arms of the converter support the whole phase current $i_{v}$ during some parts of the cycle. As the maximum current that the IGBTs can support is limited, one way to ensure that they are not overloaded is by imposing the following limits:

$$
-\bar{I}_{v} \leq i_{v} \leq \bar{I}_{v}, \forall v \in \mathcal{V}
$$

\subsection{2. $D C$ and $A C$ voltage level coupling}

Apart from the voltage limits imposed at both sides of the converter, it is necessary to take into account that the voltage level on the DC side exerts 
a limit on the maximum voltage that can be obtained at the $\mathrm{AC}$ side of the converter, [18]. This can be simplified as a ratio between the AC and DC side voltages that can be defined as (6). In this paper, the factor $k_{v}$ is set to be 1.1 as in [10]. Converters are assumed to be operated in nominal conditions. However, if other modulation mode or methods are used to obtain higher voltages for VSC AC buses, this factor could be modified accordingly [10].

$$
v_{c} \leq k_{v} \cdot v_{i} \quad \forall v \in \mathcal{V}, c \in \mathcal{N}_{v}^{a c}, i \in \mathcal{N}_{v}^{d c},
$$

\subsubsection{Maximum current through the DC cables}

This limit is to constrain the maximum current through the cables. Since the entire formulation presented in Section 3 is in [p.u.] system, and given that DC voltage does not vary very much with respect to the nominal value, the limit imposed on current thereby is equivalent to setting the maximum DC power transfer allowed as in (22).

\subsection{4. $P Q$ capability curves}

Capability curves based on active and reactive power limits are conventionally used for PF applications. Following the proposed OPF approach, there is no requirement to include the explicit capability curve limitations, as they are implicitly present in the equations included to represent the electrical network.

In addition to the above three constraints to ensure the safe operation of the converters in steady state, previous imposed limits have further derived boundaries on active and reactive power injected to the AC network [6], [10]. The reactive power $q_{v}$ injected to the $\mathrm{AC}$ side will be considered positive in case of being capacitive:

$$
p_{v}^{2}+q_{v}^{2}=\left(v_{c} \cdot i_{v}\right)^{2} \quad \forall c \in \mathcal{N}_{v}^{a c}, v \in \mathcal{V}
$$

Moreover, as explained in [19], presumably a steady state minimum DC voltage 
can exist and prohibit continuous operation while absorbing reactive power, thus a minimum of -0.5 p.u. imposed to the converters as in [10], where $\bar{Q}_{v}$ is the maximum allowed reactive power. This is also illustrated with a $\mathrm{P}-\mathrm{Q}$ diagram provided for HVDC Light of ABB [19]:

$$
q_{v} \geq \underline{q_{v}}=-\frac{\bar{Q}_{v}}{2} \quad \forall v \in \mathcal{V}
$$

Apart from the limits aforementioned, converters can be subject to certain requirement from the Grid Codes. For example, in [9], the converters are obliged to have a reactive power capacity of 0.95 power factor lagging to 0.95 leading at the connection point.

\section{Optimal Power Flow Modeling of a Hybrid AC/DC Network}

In this section, detailed mathematical formulations are presented for the proposed nonlinear programming OPF model. For simplicity, all variables presented in this section are in [p.u.], and units of corresponding parameters are adapted accordingly.

\subsection{AC Network Constraints}

\subsubsection{AC Power Flow Equations}

Every bus $i$ of the AC grid is characterized by its voltage magnitude $v_{i}$ and phase angle $\theta_{i}$. By denoting $\theta_{i j}=\theta_{i}-\theta_{j}$, power injections at node $i$ and power flows (from $i$ to $j$ ) on branch $l$, i.e., $(i, j) \in \mathcal{N}_{l}$, are [20]:

$$
\begin{gathered}
p_{i}=v_{i} \sum_{j \in \mathcal{B}^{a c}} v_{j}\left[G_{i j}^{a c} \cos \left(\theta_{i j}\right)+B_{i j} \sin \left(\theta_{i j}\right)\right], \forall i \in \mathcal{B}^{a c} \\
q_{i}=v_{i} \sum_{j \in \mathcal{B}^{a c}} v_{j}\left[G_{i j}^{a c} \sin \left(\theta_{i j}\right)-B_{i j} \cos \left(\theta_{i j}\right)\right], \forall i \in \mathcal{B}^{a c} \\
p_{l}=v_{i}^{2} G_{l}-v_{i} v_{j}\left[G_{l} \cos \left(\theta_{i j}\right)+B_{l} \sin \left(\theta_{i j}\right)\right] \\
q_{l}=-v_{i}^{2}\left(B_{l}+B_{l}^{s h t}\right)-v_{i} v_{j}\left[G_{l} \sin \left(\theta_{i j}\right)-B_{l} \cos \left(\theta_{i j}\right)\right]
\end{gathered}
$$


Notice that transformers are modeled as regular lines with pre-defined tap ratios.

Conservation of power are established through (13) and (14) taking into account active power injections from VSCs:

$$
\begin{aligned}
& p_{i}=\sum_{g \in \mathcal{G}_{i}}\left(p_{g}\right)-P_{D i}+\sum_{v \in \mathcal{V}_{i}} p_{v}^{a c}+n p_{i}, \forall i \in \mathcal{B}^{a c} \\
& q_{i}=\sum_{g \in \mathcal{G}_{i}}\left(q_{g}\right)-Q_{D i}+\sum_{v \in \mathcal{V}_{i}} q_{v}^{a c}+n q_{i}, \forall i \in \mathcal{B}^{a c}
\end{aligned}
$$

\subsubsection{AC Bus Voltage Limits}

$$
\underline{V}_{i} \leq v_{i} \leq \bar{V}_{i}, \forall i \in \mathcal{B}^{a c}
$$

\subsubsection{AC Transmission Line Capacity Limits}

$$
\sqrt{p_{l}^{2}+q_{l}^{2}} \leq \bar{S}_{l}, \forall l \in \mathcal{L}^{a c}
$$

In the computer implementation of this model, this constraint will be formulated according to the equivalent expression in order to help the non-linear optimization solver:

$$
p_{l}^{2}+q_{l}^{2} \leq \bar{S}_{l}^{2}, \forall l \in \mathcal{L}^{a c}
$$

\subsection{Network Constraints}

\subsubsection{Power Flow Equations}

Every bus $i$ of the DC grid is characterized by its voltage magnitude $v_{i}$. Every line $l$ connecting a pair of DC buses can be represented by its resistance $R_{l}$. Assuming that the extreme nodes of such line are $i$ and $j$, the real power injected at node $i$ and power flows (from $i$ to $j$ ) on line $l$ of the DC grid satisfy the following expression:

$$
\begin{gathered}
p_{i}=n \cdot v_{i} \sum_{j \in \mathcal{B}_{\mathrm{dc}}} G_{i j}^{d c}\left(v_{i}-v_{j}\right), \forall i \in \mathcal{B}^{d c} \\
p_{l}=n \cdot\left[v_{i}\left(v_{i}-v_{j}\right) / R_{l}\right],(i, j) \in \mathcal{N}_{l}
\end{gathered}
$$

In this paper, it is assumed a symmetric monopole configuration, i.e., $n=2$. VSC losses $\rho_{v}$ are incorporated for power conservation seen from DC side:

$$
p_{i}=\sum_{v \in \mathcal{V}_{i}}\left(p_{v}^{d c}+\rho_{v}\right), \forall i \in \mathcal{B}^{d c}
$$


3.2.2. DC Bus Voltage Limits

$$
\underline{V}_{i} \leq v_{i} \leq \bar{V}_{i}, \forall i \in \mathcal{B}^{d c}
$$

3.2.3. DC Transmission Line Capacity Limits

$$
-\bar{P}_{l} \leq p_{l} \leq \bar{P}_{l}, \forall l \in \mathcal{L}^{d c}
$$

\subsection{Additional VSC constraints}

As explained in previous Section 2.2, converter losses depend on whether it operates as a rectifier or an inverter, that entails the phase current are in completely opposite directions. Consequently, in order to distinguish such difference, $(23)$ is included to force converter phase current to be the same direction with the power injected to the corresponding AC bus:

$$
0 \leq i_{v} \cdot p_{v}^{a c}, \forall v \in \mathcal{V}
$$

\subsection{Generator Capacity}

In addition to network constraints and VSC modeling, there are also some generator technical limits need to fulfill:

$$
\begin{aligned}
& \underline{P}_{g} \leq p_{g} \leq \bar{P}_{g}, \forall g \in \mathcal{G} \\
& \underline{Q}_{g} \leq q_{g} \leq \bar{Q}_{g}, \forall g \in \mathcal{G}
\end{aligned}
$$

\subsection{Objective Function/Optimization Criterion}

The considered Objective Function (OF) is to minimize the total operating costs plus the penalty from the non-served active and reactive power as shown in (26) assuming $n p_{i} \geq 0, n q_{i} \geq 0 . A_{g}, B_{g}$ and $C_{g}$ are cost coefficients of generators. $N C_{p}$ and $N C_{q}$ represent the unitary costs of non-served real and reactive power respectively. There are some other alternatives, such as minimization of network losses as in [9], which could also be easily adapted.

$$
\min \sum_{g \in \mathcal{G}}\left(C_{g}+A_{g} \cdot p_{g}+B_{g} \cdot p_{g}^{2}\right)+\sum_{i \in \mathcal{B}}\left(N C_{p} \cdot n p_{i}+N C_{q} \cdot n q_{i}\right)
$$




\section{Converter Loss Impact Assessment of VSC-MTDC Systems}

In this section, an approach to evaluate the impact of converter losses on the OPF solution of AC/DC hybrid systems is proposed using the extended model described in Section 3. Firstly, it is necessary to define a comparison metric in order to compare the goodness and accuracy of the solution obtained when a simplified representation of the losses is used. Secondly, as the differences between the benchmark case and the simplified-losses cases could depend on the particular characteristic of the system under study, this paper proposes to replicate the analysis for different deployments of the HVDC grid, and for a full range of possible levels of the demand.

\subsection{Comparison Metrics}

The solution of the OPF of a hybrid AC/DC system consists of a large amount of output variables: active and reactive power injected by all the generators, voltage magnitudes and angles at every $\mathrm{AC}$ bus, active and reactive power flows at every AC line, voltage levels at the DC buses, and active power flows at every DC line. In order to compare easily the solution obtained with different degrees of simplification of converter losses modeling, it is necessary to define a few indicators that summarize how far the solution obtained with the simplified losses modeling is with respect to the benchmark case. As the objective function depends only on the active power generated by the units, and as active power flows are in general significantly higher than reactive power flows, the comparison will be carried out just in terms of differences of real power.

\subsubsection{System costs}

Given that the "Complete" modeling provides the benchmark value of the OF, the deviation of the other three modeling approaches (Avg, Prop and Lss) 
can be measured in relative terms as follows $(27)$ :

$$
\Delta O F_{\%}^{\text {Avg,Prop }, \text { Lss }}=\frac{O F^{\text {Avg,Prop }, \text { Lss }}-\text { OF Complete }}{O F^{\text {Complete }}} \times 100 \%
$$

\subsubsection{Active power flow differences}

After solving the OPF, as many power flows as number of branches will be generated. In order to measure how close the solution of the simplified methods are with respect to the "Complete" modeling, the Mean Absolute Error (MAE) is proposed. Notice that positive and negative deviations are not compensated among them. Therefore, a null MAE will be obtained only in case the power flows are exactly the same. MAE is defined as follows:

$$
\text { MAE } E^{\text {Avg,Prop }, \text { Lss }}=\frac{1}{n} \sum_{l=1}^{n}\left|f_{l}^{\text {Avg,Prop }, \text { Lss }}-f_{l}^{\text {Complete }}\right|
$$

where $n$ is the total number of observations (i.e., number of lines), $f_{l}^{\text {Avg,Prop,Lss }}$ stands for the active power flow at branch $l$ obtained with the approximated method Avg, Prop or Lss, and $f_{l}^{\text {Complete }}$ is the actual value obtained with the complete modeling. The MAE of AC and DC power flows will be calculated separately in order to identify whether the impact of converter losses modeling is more relevant in one type of network than in the other. More detailed description can be found in the Section 5.2.

\subsection{Evaluation method}

In order to take into account the dependence of the impact of converter losses on the characteristic of the power system, the the essential steps of the proposed approach can be described as follows:

- Select a set of possible hybrid AC/DC power systems $\Xi$

- For every system $\xi \in \Xi$ build a set of $K$ demand vectors $\boldsymbol{d}^{\xi}$ where each component $d_{i}^{\xi}$ represents the demand at every node $i: \boldsymbol{d}^{\xi, 1}, \ldots, \boldsymbol{d}^{\xi, k}, \ldots, \boldsymbol{d}^{\xi, K}$. 
Demand vectors $\boldsymbol{d}^{\xi, k}$ can be obtained by multiplying the nominal demand level at every node by a factor that can range from a minimum value to a maximum one with a predetermined step size. In case the demand profiles follow any particular correlation, without loss of generality, these demand scenarios could be generated applying some more sophisticated techniques.

- For every demand vector $k$ simulate the optimal operation of the power system by running the OPF model presented in this paper with the complete modeling of converter losses, and with the three simplified approaches (Avg, Prop and Lss)

- Compute the values of $\triangle O F_{\%}^{A v g, P r o p, L s s}$ and $M A E^{\text {Avg,Prop,Lss }}$

Notice that this method is completely general and could be used to compare the solution of any OPF model with respect to the accurate solution whenever it is possible to find it.

\section{Case study}

\subsection{System Description}

Fig. 4 provides the single line diagram of the two hybrid AC/DC power systems (7-Terminal and 9-Terminal that will be referred to as $7 \mathrm{~T}$ and $9 \mathrm{~T}$ ) that are going to be studied in this paper. Both systems are shown in the same diagram as they share exactly the same AC network. It is based on a Modified IEEE Two Area RTS-96 (MRTS) network where 7T consists of two separate MTDC links mainly functioning as interconnections between the two areas (blue dashed DC lines), and the 9T (red solid DC lines) has a more meshed configuration overlaying on the complete AC network. All the lines parameters 


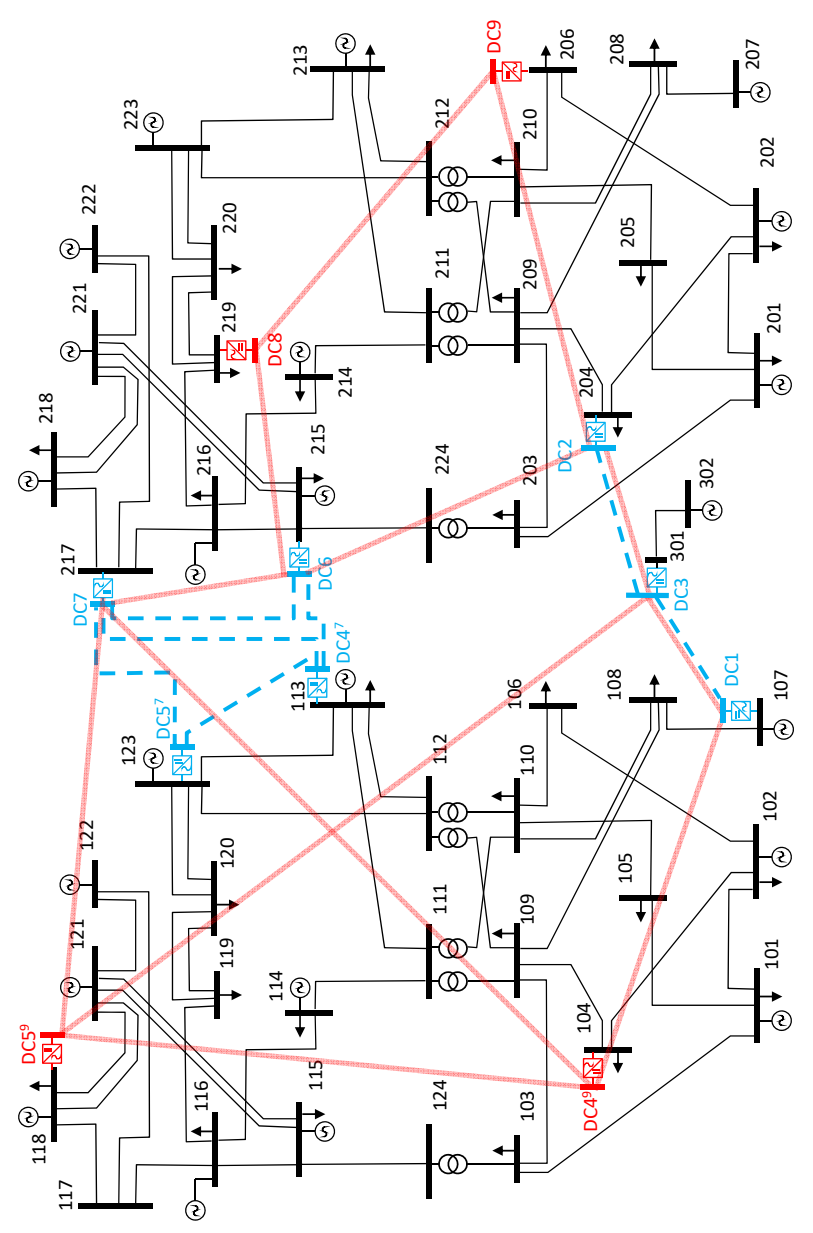

Figure 4: IEEE Two Area RTS-96 with two HVDC networks (7 and 9 terminals)

of $\mathrm{AC}$ branches can be found in [21], where the maximum capacity of the lines ranges from $175 \mathrm{MW}$ to $500 \mathrm{MW}$

Regarding the input data, 7T system is identical to the one used in [6] where the two isolated DC grids have different voltages: $\pm 300 \mathrm{kV}$ and $\pm 150 \mathrm{kV}$. In the case $9 \mathrm{~T}$, the unique $\mathrm{DC}$ grid has a nominal voltage of $\pm 300 \mathrm{kV}$. Reference buses remain unchanged. $\mathrm{DC}_{4}$ and $\mathrm{DC}_{5}$ are relocated to $\mathrm{AC}$ buses 104 and 118 respectively. In addition, two extra $\mathrm{DC}$ buses $\left(\mathrm{DC}_{8}\right.$ and $\left.\mathrm{DC}_{9}\right)$ are positioned at AC buses 219 and 206. The two corresponding converters are assigned the same 
Table 1: DC Transmission Line Data

\begin{tabular}{c|c|c|c}
\hline From DC Bus & To DC Bus & $\mathrm{R}(\mathrm{pu})$ & Flow Limit $(\mathrm{MW})$ \\
\hline \hline 1 & 3 & 0.0352 & 100 \\
\hline 1 & 4 & 0.0828 & 100 \\
\hline 2 & 3 & 0.0352 & 100 \\
\hline 2 & 6 & 0.0828 & 100 \\
\hline 2 & 9 & 0.0828 & 100 \\
\hline 3 & 5 & 0.1656 & 100 \\
\hline 4 & 5 & 0.1242 & 100 \\
\hline 4 & 7 & 0.1242 & 100 \\
\hline 5 & 7 & 0.1242 & 100 \\
\hline 6 & 7 & 0.0248 & 100 \\
\hline 6 & 8 & 0.0828 & 100 \\
\hline 8 & 9 & 0.0828 & 100 \\
\hline
\end{tabular}

parameters as the ones in $\mathrm{DC}_{6}$ and $\mathrm{DC}_{7}$ accordingly, while the others keep the same as in the $7 \mathrm{~T}$ network. DC line data for 9T network is shown in Table 1. Both case studies use same set of cost coefficients for all generators, which can be found in [22]. All power injections and voltage levels at VSCs are considered decision variables. The penalty cost for non-served active and reactive power is set to be $1000 \$ / \mathrm{MW}$ and $1000 \$ /$ Mvar respectively [23].

The ratios $\alpha$ used in (3) are calculated from computing the accurate losses at every converter, and by averaging the ratios obtained for different demand levels of the reference case and for all the converters. For each of the systems, all converters are assigned the same average value.

\subsection{Numerical Results}

The OPF model presented in this paper has been implemented in GAMS [24] on an Intel-i7 2.93GHz personal computer with 4GB of RAM memory. The OPF model considers the complete set of non-linear power flow equations. As in [10], IPOPT solver [25] has been chosen given its good performance for solving large-scale nonlinear problems. In order to validate the results, the obtained 
solution of the OPF model has been compared with the solution of the VSCMTDC power flow model MatACDC presented in [26], which is based on the Matlab toolbox MATPOWER. Fixing in MatACDC the active and reactive power of each generator to the optimal solution of the OPF, it gives exactly the same power flows and corresponding variables (voltage angles, magnitudes, etc.).

Following the approach proposed in Section 4.2, the demand scenarios have been built by multiplying the nominal demand at every node by a factor ranging from 0.6 to 1.05 with a step size of 0.05 p.u. These limits have been identified as the ones than ensure the feasibility of the optimization problem for the topologies under study, given that unit-commitment decisions are given as input data, and therefore, it is not possible to decrease the output power below certain limits, and neither to exceed a maximum power.

The economic impact (in \% of variation of the objective function) is shown in Table 2 according to the expression presented in (27). It can be seen that the impact is close to $|1| \%$ in many cases (the highest mismatches have been highlighted for each case). For instance, in the $7 \mathrm{~T}$ system, the objective functions difference reaches $0.86 \%$ with the Prop-modeling while for the $9 \mathrm{~T}$ system, such difference reaches $-0.75 \%$ with Lss-modeling. Extrapolating these percentage to a bigger system where the size of the DC grid is comparable in relative terms as the ones used in the example cases, it could be concluded that the way the converter losses are modeled can have a significant economic implication on the overall operational costs. Notice that the Lss- modeling provides lower operating costs for every demand scenario as it ignores the converter losses $\left(\Delta O F_{\%}^{L s s} \leq 0\right)$. For $A v g-$ and Prop-modeling methods it cannot be identified any particular pattern of how the values of $\Delta O F_{\%}^{A v g}$ and $\Delta O F_{\%}^{\text {Prop }}$ vary with respect to the demand. 
Table 2: O.F. deviation in Percentage [\%] (7T and 9T)

\begin{tabular}{|c|c|c|c|c|c|c|}
\hline Systems & \multicolumn{3}{|c|}{$7 \mathrm{~T}$} & \multicolumn{3}{|c|}{$9 \mathrm{~T}$} \\
\hline $\begin{array}{l}\text { Modeling } \\
\text { Method }\end{array}$ & Lss & Avg & Prop & Lss & Avg & Prop \\
\hline 0.60 & -0.25 & 0.03 & 0.86 & -0.28 & -0.13 & 0.43 \\
\hline 0.65 & -0.16 & -0.07 & -0.09 & -0.13 & 0.05 & -0.05 \\
\hline 0.70 & -0.39 & -0.20 & 0.25 & -0.11 & 0.22 & 0.13 \\
\hline 0.75 & -0.28 & 0.20 & -0.01 & -0.25 & 0.24 & 0.26 \\
\hline 0.80 & -0.31 & -0.07 & -0.04 & -0.41 & 0.42 & 0.04 \\
\hline 0.85 & -0.23 & 0.26 & 0.05 & -0.24 & 0.12 & 0.23 \\
\hline 0.90 & -0.22 & 0.65 & 0.05 & -0.33 & 0.30 & 0.14 \\
\hline 0.95 & -0.26 & 0.50 & 0.45 & -0.44 & -0.14 & 0.74 \\
\hline 1.00 & -0.69 & -0.04 & 0.08 & -0.75 & -0.03 & 0.21 \\
\hline 1.05 & -0.53 & 0.10 & 0.24 & -0.25 & 0.11 & 0.61 \\
\hline
\end{tabular}

Regarding the impact on power flows, AC and DC lines are analyzed separately for each modeling method by comparing their MAE according to (28). The results of $7 \mathrm{~T}$ and $9 \mathrm{~T}$ systems are plotted together by pairs for each demand factor value. For instance, the dash-dotted rectangular box in Fig. 5 shows the boxplots for the $7 \mathrm{~T}$ (on the left) and 9T (on the right) systems for the demand level of 0.6. Figures 5 and 6 present the boxplots of the absolute differences of power flows at every AC and DC line respectively for every demand level. For a given box-plot, the straight horizontal line in red represents the obtained MAE for all lines in that demand level scenario. In this paper, the widely acknowledged definition of boxplot, also known as box-whisker diagram, is adopted [27], [28]. The blue box contains $50 \%$ of the data set once the outliers (marked as "+" in red) have been discarded (i.e. lower and upper boundaries are 25th and $75 t h$ percentiles). Tables 3 and 4 provide the numerical values.

It is important to highlight that for both $7 \mathrm{~T}$ and $9 \mathrm{~T}$ systems, $\mathrm{AC}$ branch power flows differences are in general smaller when compared to DC branches. In addition, $\mathrm{AC}$ flows MAE are higher $70 \%$ of the studied cases in the $9 \mathrm{~T}$ system. However, for the DC flows the behavior is the opposite one: only $10 \%$ of the 


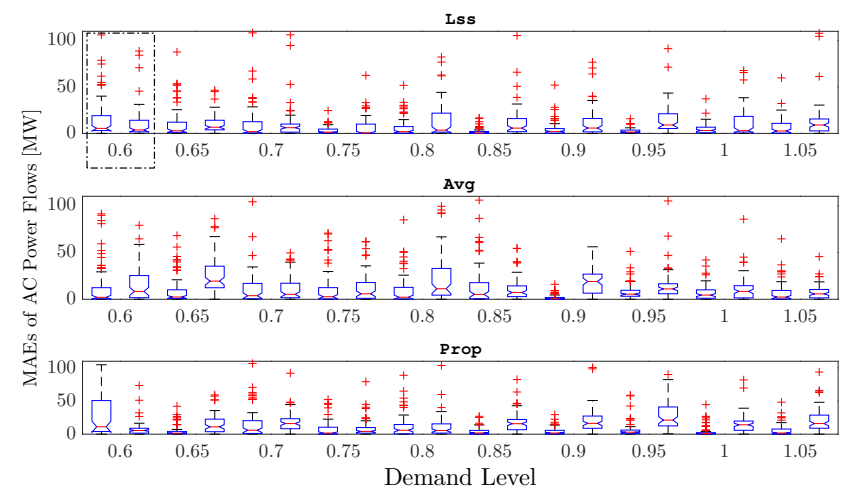

Figure 5: MAE of AC Power Flows [MW] (7T/9T)

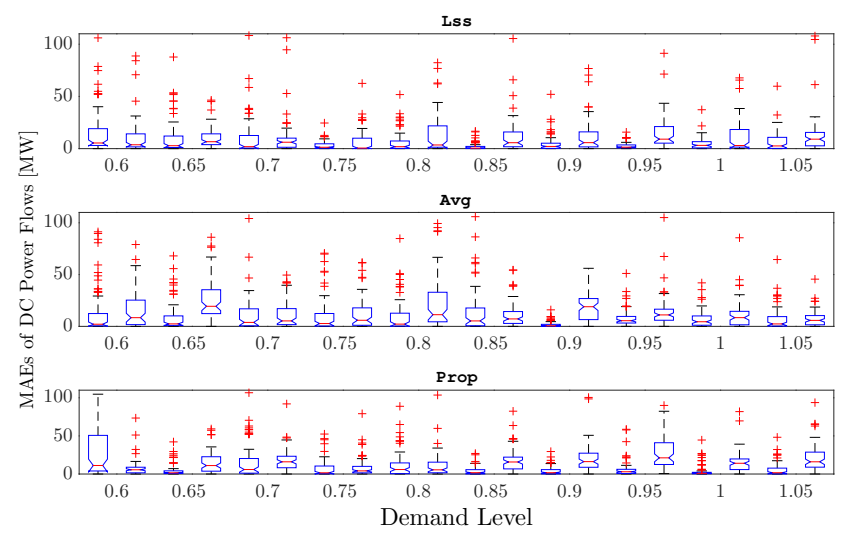

Figure 6: MAE of DC Power Flows [MW] (7T/9T)

Table 3: MAE of AC Power Flows [MW]

\begin{tabular}{c|r|r|r|r|r|r}
\hline Systems & \multicolumn{3}{|c|}{$7 \mathrm{~T}$} & \multicolumn{3}{|c}{$9 \mathrm{~T}$} \\
\hline $\begin{array}{c}\text { Modeling } \\
\text { Method }\end{array}$ & Lss & Avg & Prop & Lss & Avg & Prop \\
\hline \hline 0.60 & 16.98 & 13.42 & 23.94 & 13.81 & 16.30 & 12.70 \\
\hline 0.65 & 10.29 & 9.33 & 4.74 & 10.18 & 17.07 & 13.24 \\
\hline 0.70 & 9.96 & 10.79 & 17.18 & 9.83 & 11.95 & 14.35 \\
\hline 0.75 & 2.95 & 11.13 & 7.42 & 6.65 & 12.84 & 11.54 \\
\hline 0.80 & 6.28 & 9.32 & 11.38 & 12.91 & 17.96 & 14.80 \\
\hline 0.85 & 2.08 & 13.25 & 3.81 & 11.70 & 11.64 & 14.20 \\
\hline 0.90 & 4.61 & 1.82 & 3.98 & 13.31 & 16.14 & 15.51 \\
\hline 0.95 & 2.59 & 7.79 & 6.11 & 15.20 & 13.36 & 17.68 \\
\hline 1.00 & 4.74 & 7.38 & 3.89 & 12.98 & 12.34 & 13.92 \\
\hline 1.05 & 6.53 & 7.00 & 5.81 & 12.98 & 9.66 & 15.37 \\
\hline \hline Mean & $\mathbf{6 . 7 0}$ & $\mathbf{9 . 1 0}$ & $\mathbf{8 . 8 2}$ & $\mathbf{1 1 . 9 6}$ & $\mathbf{1 3 . 9 3}$ & $\mathbf{1 4 . 3 3}$ \\
\hline
\end{tabular}


Table 4: MAE of DC Power Flows [MW]

\begin{tabular}{c|c|c|c|c|c|c}
\hline Systems & \multicolumn{3}{|c|}{$7 \mathrm{~T}$} & \multicolumn{3}{c}{$9 \mathrm{~T}$} \\
\hline $\begin{array}{c}\text { Modeling } \\
\text { Method }\end{array}$ & Lss & Avg & Prop & Lss & Avg & Prop \\
\hline \hline 0.60 & 52.42 & 89.23 & 65.57 & 20.67 & 20.55 & 17.15 \\
\hline 0.65 & 65.24 & 58.85 & 26.69 & 13.53 & 21.51 & 16.35 \\
\hline 0.70 & 57.64 & 46.73 & 71.61 & 18.00 & 15.81 & 16.17 \\
\hline 0.75 & 20.98 & 63.30 & 49.05 & 13.32 & 17.32 & 12.89 \\
\hline 0.80 & 30.92 & 46.84 & 52.20 & 20.89 & 23.70 & 20.25 \\
\hline 0.85 & 12.56 & 73.98 & 18.46 & 18.99 & 15.48 & 16.65 \\
\hline 0.90 & 28.23 & 10.83 & 19.99 & 21.05 & 21.34 & 19.90 \\
\hline 0.95 & 13.16 & 33.31 & 36.70 & 20.15 & 17.70 & 23.73 \\
\hline 1.00 & 17.88 & 28.78 & 26.91 & 20.98 & 16.93 & 17.72 \\
\hline 1.05 & 35.14 & 29.59 & 25.75 & 18.16 & 11.64 & 18.29 \\
\hline \hline Mean & $\mathbf{3 3 . 4 2}$ & $\mathbf{4 8 . 1 5}$ & $\mathbf{3 9 . 2 9}$ & $\mathbf{1 8 . 5 7}$ & $\mathbf{1 8 . 2 0}$ & $\mathbf{1 7 . 9 1}$ \\
\hline
\end{tabular}

studied cases the DC flows MAE is higher for the $9 \mathrm{~T}$ system. Therefore, for these study cases it could be concluded that the more meshed the DC grid is, the higher (lower) the impact on AC (DC) flows are due to not modeling the converter losses in an accurate manner.

Another interesting finding is that Lss-modeling outperforms other two methods for $\mathrm{AC}$ branches in both systems (the average MAE values 6.70 and 11.96 are lower than the ones of Avg and Prop approaches). However, for DC branches, Lss-modeling only outperforms for the $7 \mathrm{~T}$ case. For the $9 \mathrm{~T}$ case the best approach is the proportional method. What is more important is that in all these cases, the power flow differences of DC branches (in MW) are very large considering their maximum capacities. For instance, even for the lowest average mismatch case (the Prop-modeling for the 9T system), the value 17.91 MW is very relevant taken into account that the capacity of the lines is $100 \mathrm{MW}$ (see Table 1). 


\section{Conclusions}

This paper proposes an extended OPF model for steady-state analysis of hybrid $\mathrm{AC} / \mathrm{DC}$ systems including all non-linearities of power flow equations and a detailed converter modeling including losses. In particular, converter losses have been modeled in the most accurate way according to the state-of-the-art, and three alternative approaches (lossless, proportional and average) have also been implemented. As the impact assessment can depend on the level of deployment of the DC grid, two systems have been studied: one with only interconnecting DC lines (7T system) and another one with a very meshed configuration (9T system). The evaluation method along with a comparison metric consisting of two indicators are proposed and illustrated. Obtained results show that the OPF solution is highly dependent on how converter losses are modeled. From the study case, it can be concluded that when the system is not heavily meshed, the lossless approach is the best way if complete modeling of converter losses is not possible. However, for meshed DC grids, none of the considered simplified approaches outperforms when analyzing the $\mathrm{AC}$ and $\mathrm{DC}$ power flow mismatches. The main conclusion that can be drawn from this paper is that not modeling the converter losses in an appropriate manner could lead to very different power flow values compared to the accurate formulation, especially for the DC branches. Apart from the pure operational point of view, this issue should be taken into account when planning the expansion of future MTDC networks.

\section{Acknowledgment}

This work has been carried out under the financial support of the European Project BEST PATHS within the $7^{\text {th }}$ framework programme and the SUPERRED project financed by the Spanish Ministry of Economy, Industry and 
Competitiveness and European Regional Development Fund (ENE2015-67048-

C4-2-R/MINECO/FEDER).

\section{Reference}

[1] D. Van Hertem and M. Ghandhari, "Multi-terminal vsc hvdc for the european supergrid: Obstacles," Renewable and Sustainable Energy Reviews, vol. 14, no. 9, pp. 3156-3163, Dec. 2010.

[2] A. Arapogianni, J. Moccia, D. Williams, and J. Phillips, "Wind in our Sails - The coming of Europe's offshore wind energy industry," The European Wind Energy Association, Tech. Rep., Nov. 2011.

[3] "Roadmap to the supergrid technologies. update report," Friends of the Supergrid (FOSG), Tech. Rep., Mar. 2013.

[4] K. Meah and S. Ula, "Comparative Evaluation of HVDC and HVAC Transmission Systems," in IEEE Power Engineering Society General Meeting, 200\%, Jun. 2007, pp. 1-5.

[5] N. Flourentzou, V. Agelidis, and G. Demetriades, "Vsc-based hvdc power transmission systems: An overview," IEEE Transactions on Power Electronics, vol. 24, no. 3, pp. 592-602, Mar. 2009.

[6] J. Beerten, S. Cole, and R. Belmans, "Generalized steady-state vsc mtdc model for sequential ac/dc power flow algorithms," IEEE Transactions on Power Systems, vol. 27, no. 2, pp. 821-829, May 2012.

[7] "VSC TRANSMISSION," CIGRE Working Group B4.37, Technical Report Ref.269, Apr. 2005.

[8] M. Baradar, M. Hesamzadeh, and M. Ghandhari, "Second-order cone programming for optimal power flow in vsc-type ac-dc grids," IEEE Transactions on Power Systems, vol. 28, no. 4, pp. 4282-4291, Nov. 2013.

[9] J. Cao, W. Du, H. Wang, and S. Bu, "Minimization of Transmission Loss in Meshed AC/DC Grids With VSC-MTDC Networks," IEEE Transactions on Power Systems, vol. 28, no. 3, pp. 3047-3055, Aug. 2013.

[10] W. Feng, A. L. Tuan, L. Tjernberg, A. Mannikoff, and A. Bergman, "A new approach for benefit evaluation of multiterminal vsc-hvdc using a proposed mixed ac/dc optimal power flow," IEEE Transactions on Power Delivery, vol. 29, no. 1, pp. 432-443, Feb. 2014.

[11] F. Desta Zahlay, L. Olmo, M. Rivier, F. de Cuadra, and J. I. Perez-Arriaga, "Finding a representative network losses model for large-scale transmission expansion planning with renewable energy sources," Energy, Dec. 2015. 
[12] B. K. Bose, Power Electronics and Motor Drives: Advances and Trends. Academic Press, Jul. 2010.

[13] S. Rohner, S. Bernet, M. Hiller, and R. Sommer, "Modulation, Losses, and Semiconductor Requirements of Modular Multilevel Converters," IEEE Transactions on Industrial Electronics, vol. 57, no. 8, pp. 2633-2642, Aug. 2010 .

[14] Z. Zhang, Z. Xu, and Y. Xue, "Valve losses evaluation based on piecewise analytical method for MMC-HVDC links," IEEE Transactions on Power Delivery, vol. 29, no. 3, pp. 1354-1362, Jun. 2014.

[15] U. N. Gnanarathna, A. M. Gole, A. D. Rajapakse, and S. K. Chaudhary, "Loss Estimation of Modular Multi-Level Converters using ElectroMagnetic Transients Simulation," in Proc. Int. Conf. Power Syst. Transient (IPST), 2011.

[16] C. Oates and C. Davidson, "A comparison of two methods of estimating losses in the Modular Multi-Level Converter," in Proceedings of the 201114 th European Conference on Power Electronics and Applications (EPE 2011), Aug. 2011, pp. 1-10.

[17] "Guide for the Development of Models for HVDC Converters in a HVDC Grid," CIGRE Working Group B4.57, Tech. Rep., Dec. 2014.

[18] S. Cole, "Steady-state and dynamic modelling of VSC HVDC systems for power system Simulation," Ph.D. dissertation, $\mathrm{PhD}$ dissertation, Katholieke University Leuven, Belgium, 2010.

[19] P. K. M. Vormedal, "Voltage Source Converter Technology for Offshore Grids: Interconnection of Offshore Installations in a Multiterminal HVDC Grid using VSC," M. Sc. Thesis, 2010.

[20] A. J. Wood and B. F. Wollenberg, Power Generation, Operation, and Control, 2nd ed. John Wiley \& Sons, Nov. 2012.

[21] P. Wong, P. Albrecht, R. Allan, R. Billinton, Q. Chen, C. Fong, S. Haddad, W. Li, R. Mukerji, D. Patton, A. Schneider, M. Shahidehpour, and C. Singh, "The IEEE reliability test system-1996. a report prepared by the reliability test system task force of the application of probability methods subcommittee," IEEE Transactions on Power Systems, vol. 14, no. 3, pp. 1010-1020, Aug. 1999.

[22] P. Subcommittee, "IEEE Reliability Test System," IEEE Transactions on Power Apparatus and Systems, vol. PAS-98, no. 6, pp. 2047-2054, Nov. 1979.

[23] F. Leveque, Ed., Competitive Electricity Markets And Sustainability. Edward Elgar Pub, Feb. 2007. 
[24] A. Brooke, D. Kendrick, and A. Meeraus, GAMS: A User's Guide. Redwood City, California: The Scientific Press, 1992.

[25] "GAMS - The Solver Manuals," Oct. 2015. [Online]. Available: http://www.gams.com/help/topic/gams.doc/solvers/allsolvers.pdf

[26] J. Beerten, S. Cole, and R. Belmans, "A sequential ac/dc power flow algorithm for networks containing multi-terminal vsc hvdc systems," in Power and Energy Society General Meeting, 2010 IEEE, Jul. 2010, pp. 1-7.

[27] D. Zwillinger and S. Kokoska, CRC Standard Probability and Statistics Tables and Formulae. CRC Press, Dec. 1999.

[28] G. Upton and I. Cook, Understanding Statistics. Oxford University Press, 1996. 